\title{
Application of Differential Evolution to Passive Shunt Harmonic Filter Planning
}

\author{
Tien-Ting Chang and Hong-Chan Chang
}

\author{
Department of Electrical Engineering, National Taiwan University of Science and Technology
}

Taipei, Taiwan, 10672, R.O.C.

\begin{abstract}
This paper presents a refined differential evolution (RDE) for passive shunt harmonic filter planning. The purpose is to minimize total costs while satisfying various practical constraints. The substation harmonic voltage sources and load harmonic current sources are considered simultaneously. In addition, practical constraints such as the voltage magnitude limit, total harmonic distortion and the commercially available discrete sizes of the capacitors can be accounted for. The RDE approach together with the evolutionary programming (EP) were tested on a 9-bus distribution system. Results obtained show that the proposed RDE method can provide a highly optimal solution within a reasonable time.
\end{abstract}

Keywords: passive shunt harmonic filter, total harmonic distortion, differential evolution.

\section{INTRODUCTION}

Harmonic currents originating from any nonlinear load may flow into a power system. These harmonic currents can cause voltage distortion and excessive power losses in the system, hence operating problems in power system arise. In the past, several methods have been suggested to diminish harmonics problem such as series or shunt active power filters. However, in practice, tuned passive filters are widely employed due to their simplicity and economical cost [1]. Installation of tuned passive filters in a power system is a very complicated problem, for instance, the harmonic standard, locations and sizes of tuned passive filters, power losses, as well as filter costs must be thoroughly considered. In addition, the sizes of the commercially available capacitors are discrete in nature and the cost of per kvar at each size is quite different [2]. Because the $L$ and $C$ values at the single tuned $L C$ filters are dependent, the size of the filters is discrete in the same way as the capacitors. In fact, the principal harmonic components occurring in the distribution system are the lower order frequencies such as the third, fifth and seventh

Paper accepted for presentation at the $\delta^{\text {sh International }}$ Conference on Harmonics and Quality of Power ICHQP '98, jointly organized by IEEE/PES and NTUA, Athens, Greece, October 14-16, 1998

0-7803-5105-3/98/\$ 10.00 @ 1998 IEEE harmonics [3-6]. Thus, emphasis is placed on the study of these lower order frequencies in harmonic filter planning.

Over the past few years, a considerable number of methods have been proposed for reduction of harmonic levels. For example, simulated annealing by LC compensators was reported to reduce harmonic levels in a distribution system [7]. In [8], an approach including a two-step procedure for distribution system harmonic filter planning was proposed. The problem of passive shunt harmonic filter planning is a combinatorial optimization problem with equality and inequality constraints, which includes limits on the rms values and the total harmonic distortion of the bus voltages. The purpose of this paper is to present a refined differential evolution to effectively solve the harmonic filter planning problem. Differential evolution is a new heuristic approach for minimizing possibly nonlinear and non-differential functions. It is demonstrated that the DE method converges faster and with more certainty than many other acclaimed global optimization methods [9]. With several salient features implemented into the conventional DE method, the proposed approach (named $\mathrm{RDE}$ ) can effectively solve the problem of passive shunt harmonic filter planning.

\section{PROBLEM FORMULATION}

The problem of passive shunt harmonic filter planning is to determine the locations, types, and sizes of LC tuned filters. The objective is to minimize the sum of the facility installation and operational costs (power losses) while satisfying the constraints of the bus voltages and total harmonic distortion. Passive shunt harmonic filter planning can be formulated as a combinatorial optimization problem as given below:

\section{A. Objective Function}

The objective function of the problem can be expressed as follows:

$$
\text { Min } C_{\text {total }}=\left(C_{P_{L}}+C_{F}\right)
$$

where

$$
C_{P_{l}}=K_{p} \sum_{k} T_{k} \sum_{i=1}^{n_{s}} \sum_{h=1}^{n_{h}} I_{i h}^{2} R_{i}
$$




$$
C_{F}=\sum_{i=1}^{n_{h}} \sum_{j \in F_{s}} K_{i j} Q_{i j}
$$

and

$C_{P_{L}}=$ the cost of total real power loss,

$C_{F}=$ the cost of installation of LC tuned filters,

$k=\{1,2,3, \ldots\}$ is the set of load levels,

$F_{s}=\{3,5,7,11, \ldots\}$ is the filter order,

$n_{h}=$ the total harmonic order,

$n_{s}=$ the total number of system sections,

$n_{b}=$ total number of buses in the system,

$I_{i h}=$ the current for harmonic $h$ flowing in the $i^{\text {th }}$ section,

$R_{i}=$ the resistance of the $i^{\text {th }}$ section,

$K_{P}=$ the cost of per unit power loss, in $\$ / \mathrm{kWh}$,

$Q_{i j}=$ the size of the $j^{\text {th }}$ harmonic filter at bus $i$, in kvar

$K_{i j}=$ the cost per kvar corresponding to the size $Q_{i j}$, in $\$ /$ kvar.

\section{B. Equality and Inequality Constraints}

The equality constraints are the real and reactive power flow equations. The sizes of the LC tuned filters are discrete values. In this study, the power flow equations in the radial system are solved by recursive equations [7]. The models of the feeder and load in [2] are employed:

$$
\begin{aligned}
& z_{i}^{h}=R_{i}+j h X_{i} \quad, i=1,2, \ldots n_{s} \\
& y_{l i}^{h}=\frac{P_{l i}}{\left|Y_{i}^{1}\right|^{2}}-j \frac{Q_{l i}}{h\left|Y_{i}^{1}\right|^{2}} \quad, i=1,2, \ldots n_{b}
\end{aligned}
$$

where

$z_{i}^{h}=$ the impedance of the $i^{\text {th }}$ section,

$X_{i}=$ the inductive reactance of the $i^{\text {th }}$ section.

For a single tuned LC filter, assuming a filter resonated at $h_{i}$ harmonic frequency, the relation between the inductor and capacitor can be derived as follows:

$$
\begin{aligned}
& Q_{i}^{C}=\omega C_{i} V^{2} \\
& Q_{i}^{L}=\frac{V^{2}}{\omega L_{i}} \\
& h_{i}=\sqrt{1 / \omega^{2} L_{i} C_{i}}=\sqrt{Q_{i}^{L} / Q_{i}^{C}} \\
& h_{i} \in\{3,5,7,11, \ldots\}
\end{aligned}
$$

where

$Q_{i}^{C}=$ the size of the capacitor $C_{i}$ at bus $i$,

$Q_{i}^{L}=$ the size of the inductor $L_{i}$ at bus $i$.

Thus, the size of the filter and the corresponding impedance at the $h^{\text {th }}$ harmonic frequency are

$$
Q_{i j}=\frac{h_{i}^{2} Q_{i}^{C}}{h_{i}^{2}-1}
$$

and

$$
Z_{i}^{h}=j\left(h^{2}-h_{i}^{2}\right) S_{b} / h\left(h_{i}^{2}-1\right) Q_{i j} .
$$

Because the commercially available capacitor sizes are integer multiples of the smallest standard size $Q_{0}$, $Q_{i}^{C}$ and $Q_{i j}$ can be expressed as follows:

$$
\begin{aligned}
& Q_{i}^{C} \in\left\{Q_{0}, 2 Q_{0}, \ldots, m Q_{0}\right\} \\
& Q_{i j} \in\left\{Q_{j 0}, 2 Q_{j 0}, \ldots, m Q_{j 0}\right\}
\end{aligned}
$$

and

$$
K_{i j} \in\left\{K_{j c 1}, K_{j c 2}, \ldots, K_{j c m}\right\}
$$

where

$Q_{j 0}=$ the smallest size of the $j^{\text {th }}$ harmonic filter, in kvar,

$m Q_{j 0}=$ the maximum size of the $j^{\text {th }}$ harmonic filter, in kvar,

$K_{j c 1}=$ the cost of per kvar corresponding to the size $Q_{j 0}$, in $\$ / \mathrm{kvar}$.

The inequality constraints require that the voltage magnitude and the total harmonic distortion be within the specified limits.

$$
\begin{array}{ll}
V_{\min } \leq\left|V_{i}\right| \leq V_{\max } & , i=1,2, \ldots n_{b} \\
T H D_{i} \leq T H D_{\max } & , i=1,2, \ldots n_{b}
\end{array}
$$$$
\text { and }
$$

where

$$
V_{i}=\sqrt{\sum_{h=1}^{n_{h}}\left(V_{i}^{h}\right)^{2}}
$$

$\left[\begin{array}{c}V_{1}^{h} \\ V_{2}^{h} \\ \vdots \\ V_{n_{b}}^{h}\end{array}\right]=\left[\begin{array}{cccc}z_{11}^{h} & z_{12}^{h} & \cdots & z_{1 n_{b}}^{h} \\ z_{21}^{h} & z_{22}^{h} & \cdots & z_{2 n_{h}}^{h} \\ \vdots & \vdots & \cdots & \vdots \\ z_{n_{b} 1}^{h} & z_{n_{b} 2}^{h} & \cdots & z_{n_{b} n_{b}}^{h}\end{array}\right]\left[\begin{array}{c}I_{1}^{h} \\ I_{2}^{h} \\ \vdots \\ I_{n_{b}}^{h}\end{array}\right]$

$$
T H D_{i}=\frac{\sqrt{\sum_{h \neq 1}\left(V_{i}^{h}\right)^{2}}}{\left|V_{i}^{1}\right|}
$$

\section{where}

$V_{\min }, V_{\max }=$ the specified voltage magnitude limits,

$T H D_{\max }=$ the specified voltage total harmonic distortion limit,

$Z_{i j}^{h}=$ the element of the bus impedance at the

$h^{\text {th }}$ harmonic frequency,

$I_{i}^{h}=$ the equivalent harmonic current source at bus $i$.

\section{THE REFINED DIFFERENTIAL EVOLUTION}

DE is a parallel direct search method, whose main procedures are initialization, mutation, crossover, and selection. The initial population is randomly selected and should cover the entire parameter space. The mutant 
vectors are generated by adding the weighted difference between two target vectors. Then, the parameters of the mutant vector and the target vector are mixed to yield the trial vector. If the trial vector induces a smaller cost function value than the target vector, the trial vector replaces the target vector in the following generation.

The DE procedure for passive shunt harmonic filter planning is briefly summarized as follows:

(1) Initialization: The initial vector population is chosen by randomly selection

$$
Q_{i}^{0}=U\left(0, Q_{\max }\right)^{k} \quad, i=1,2, \ldots n p
$$

$Q_{i}^{0}$ is the size vector of the LC tuned filters, $U\left(0, Q_{\max }\right)^{k}$ denotes a uniform random number generator ranging over $\left[0, Q_{\max }\right]$ in each of $k$ dimensions and $n p$ is the population size.

(2) Mutation: A mutant vector is generated in the mutation process at the $G^{\text {th }}$ generation according to

$$
\hat{Q}_{i}^{G+1}=Q_{r_{1}}^{G}+F\left(Q_{r_{2}}^{G}-Q_{r_{3}}^{G}\right) \quad i=1,2, \ldots n p
$$

where $r_{1}, r_{2}, r_{3}$ are random indexes $\in\{1,2, \ldots, n p\} . F$ is a real and constant factor $\in[0,2]$.

(3) Crossover: In order to increase the diversity of the vectors, the parameters of the mutant vector and the target vector are mixed to yield the trial vector:

$$
\bar{Q}_{i}^{G+1}=\left(\bar{Q}_{i 1}^{G+1}, \bar{Q}_{i 2}^{G+1}, \ldots, \bar{Q}_{i k}^{G+1}\right)
$$

where

$$
\begin{aligned}
& \bar{Q}_{i j}^{G+1}= \begin{cases}\hat{Q}_{i j}^{G+1} & \text { if }(\operatorname{randb}(j)) \leq C R), \text { or } j=\operatorname{rnbr}(i) \\
Q_{i j}^{G} & \text { otherwise ; }\end{cases} \\
& i=1,2, \ldots n p ; j=1,2, \ldots k
\end{aligned}
$$

In Eq. (23), $\mathrm{CR}$ is the crossover constant $\in[0,1]$. $\operatorname{randb}(j)$ is the $j^{\text {th }}$ evaluation of a uniform random number generator ranging over $[0,1]$, and $m b r(i)$ is an index randomly chosen from $\{1,2, \ldots, k\}$.

(4) Selection: The cost function values of each target vector and trial vector are obtained by running the harmonic power flow. If vector $Q_{i}^{G}$ yields a larger cost function value than $\bar{Q}_{i}^{G+1}$, then $Q_{i}^{G+1}$ is set to $\bar{Q}_{i}^{G+1}$; otherwise, the old value $Q_{i}^{G}$ is retained in the next generation.

In the refined RDE method, some modifications are made to $\mathrm{DE}$ for our planning problem:

(1) In the initialization process, we first search for the sensitive nodes that are effective in reducing the losses in the distribution system. Filters are then installed at the most sensitive node until the losses cannot be further reduced, then the next sensitive node is considered for filter installation. The procedure is repeated until no further reduction in system losses can be achieved, so that an initial vector can be determined. While the other $n p-l$ initial vectors be generated by the uniform random number generator. The modified initialization process can help in narrowing down the space of possible solutions, and thus speeds up the convergence.
(2) In the mutation process, the diversity of the target vectors is small, which means the mutant vector is close to the target vector, and may lead to a premature convergence. In order to increase the diversity of the vectors, an independent noise term can be added as follows:

$$
\hat{Q}_{i}^{G+1}=Q_{r_{1}}^{G}+F\left(Q_{r_{2}}^{G}-Q_{r_{3}}^{G}\right)+\text { noise term }
$$

and

$$
\text { noise term }=k_{\text {noise }} U(-\Delta Q, \Delta Q)
$$

where $k_{\text {noise }}$ is the noise factor. If the system losses are decreasing and the constraints are satisfied, then $k_{\text {noise }}$ is set at zero; otherwise it is set at $1 . U(-x, x)$ is a uniform random number generator ranging over $[-x, x]$.

(3) To speed up the convergence of the vectors and obtain the global optimum solution, the objective function should be extended to consider the constraints. To this end, we define two functions as below:

$$
\begin{aligned}
& g_{1}(x, Q)=C_{\text {total }}(x, Q)+w_{v} \sum_{i=1}^{n_{b}}\left(V_{i}-1\right)^{2}+w_{\text {thd }} \times \\
& \sum_{i=1}^{n_{h}}\left(T H D_{i}-T H D_{\max }\right) \\
& g_{2}(x, Q)=\sum_{i=1}^{n_{s}} \sum_{h=1}^{n_{h}} I_{i h}^{2} R_{i}
\end{aligned}
$$

where $w_{v}$ is the penalty factor for voltage magnitude limits. If the voltage magnitude constraints are violated, then a large number is given, otherwise $w_{v}$ is set at zero. Similarly, $w_{\text {thd }}$ is the penalty factor of the total harmonic distortion limit. The total real power loss is the sum of the loss in each section of the distribution system and is a function of the LC tuned filter placements. For each target vector and trial vector at every generation, compute the $g_{1}$ and $g_{2}$ values. Then the vectors are ranked in descending order corresponding to the values of $g_{1}$ and $g_{2}$, separately. The first $0.5 n p$ vectors are selected to be the target vectors at the next generation.

(4) Convergence criterion: when the specified number of generations is reached, or the best target vector cannot be improved after a specified number of generations, the DE process is stopped.

With the modification mentioned above, the robust characteristics of the DE can still be kept, and more importantly, the chance of finding the optimal solution be considerably increased with reasonable solution times.

\section{SIMULATION RESULTS AND DISCUSSION}

The proposed method was applied to a test system [2], whose feeder and load data are listed in the Appendix. The radial distribution system included 9 load buses with rated voltage at $23 \mathrm{kV}$. In addition, three load levels were considered, as shown in TABLE 1. The substation had harmonic voltage sources whose contents were $3.5 \%, 2.0 \%$, 
$1.9 \%, 1.7 \%, 1.5 \%$ for the fifth, seventh, eleventh, thirteenth, and seventeenth harmonics respectively [7]. In addition, the load buses 5,7 , and 9 had harmonic current sources, as shown in TABLE 2. The commercial three-phase capacitor sizes and the corresponding costs can be found in [2]. The cost per unit power loss $K_{P}=0.0192 \$ / \mathrm{kWh}$. In this study, the base was $23 \mathrm{kV}, 10 \mathrm{MVA}$, the voltage magnitude limit $V_{\max }=1.1$ p.u., and the voltage total harmonic distortion limit $T H D_{\max }=5 \%$.

In the original system (abbreviated "O"), the total cost, total power loss, voltage profile and voltage total harmonic distortion of each bus could be obtained by fundamental frequency power flow as well as harmonic analysis. The numerical results are listed in TABLE 3. Apparently, although the maximum voltage limit was satisfied, the total harmonic distortion and the smallest voltage magnitude violated the limits.

In order to illustrate the application of differential evolution to passive shunt harmonic filter planning, four cases were studied: (i) Case A - the total harmonic distortion constraint was ignored and only the capacitor compensation was considered, (ii) Case B - the same as Case $\mathrm{A}$, but with the total harmonic distortion constraint was taken into account, (iii) Case C - installation of harmonic filter using the RDE method, the constraints and limits were taken into account, using the RDE method, (iv) Case D - the same consideration as in Case $C$ except that the EP method was adopted.

TABLE 3 shows the results of the original system and the four cases studied. TABLE 4 indicates the locations and the sizes of the capacitors or the filters for each case.

In Case A, because the voltage total harmonic constraint was neglected, the VTHD at all buses violated the constraint value of $5 \%$, except for bus 1 . Note that the total cost and total power loss were smaller compared with the original system.

In Case B, the total cost and total power loss were larger compared with the original system, although the maximum VTHD limit was hardly satisfied.

Comparison results between Case C and Case D show that both the RDE method and the EP method are suitable for solving this problem. However, the RDE method is more likely to provide the optimal solution within a reasonable time.

Fig. 1 shows the frequency scan of the driving point impedance for bus $9\left(z_{99}\right)$ at a light load. It indicates that in Case A, if the maximum VTHD constraint is disregarded, and the capacitors compensation is considered, resonance conditions can occur at the $6^{\text {th }}$ and $13^{\text {th }}$ order harmonic frequency, where the voltage total harmonic distortions are $12.65 \%$.

Fig. 2 shows the voltage total harmonic distortion at the buses for the four cases. As indicated, if only the capacitors are considered, the maximum VTHD constraint could be violated and the maximum VTHD may occur at the end feeder.

Fig. 3 shows the rms values of the voltage at each bus at peak load. It is obvious that in the cases where filters are considered, the voltage profile can be improved significantly.

TABLE 1

DATA ON LOAD LEVELS

\begin{tabular}{|c|c|c|c|}
\cline { 2 - 4 } \multicolumn{1}{c|}{} & $\begin{array}{c}\text { Peak Load } \\
\left(T_{1}\right)\end{array}$ & $\begin{array}{c}\text { Normal Load } \\
\left(T_{2}\right)\end{array}$ & $\begin{array}{c}\text { Light Load } \\
\left(T_{3}\right)\end{array}$ \\
\hline Demand Factor & 1.5 & 1 & 0.5 \\
\hline Time Intervals(hour) & 1000 & 6760 & 1000 \\
\hline Lower Voltage Limits (pu) & 0.75 & 0.85 & 0.95 \\
\hline
\end{tabular}

TABLE 2

HARMONIC CURRENTS OF LOADS

$\left(10^{-3}\right.$ p.u. $)$

\begin{tabular}{|c|c|c|c|c|c|}
\hline bus no. & 5th & 7 th & 11 th & 13 th & 17 th \\
\hline 5 & 5.82 & 4.00 & 2.21 & 1.73 & 1.06 \\
\hline 7 & 4.44 & 3.06 & 1.90 & 1.32 & 0.81 \\
\hline 9 & 5.71 & 3.92 & 2.35 & 1.70 & 1.04 \\
\hline
\end{tabular}

TABLE 3

SUMMARY OF SIMULATED RESULTS

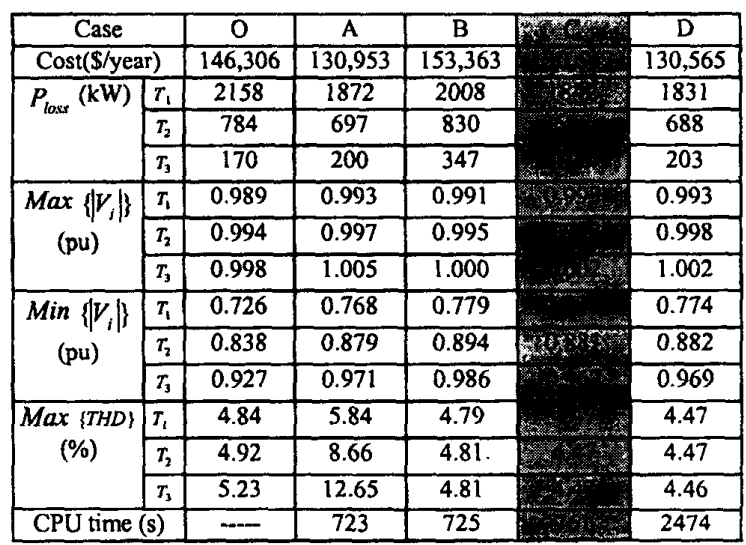

TABLE 4

OPTIMAL SOLUTION FOR EACH CASE

\begin{tabular}{|c|c|c|c|}
\hline Case & LC or C & Location & Size(kvar) \\
\hline A & Capacitor & $4,5,9$ & $2850,1200,450$ \\
\hline B & Capacitor & 4,9 & 150,2700 \\
\hline C & $5^{\text {th }}$ filter & $4,5,9$ & $3300,1200,450$ \\
\hline D & $5^{\text {hi }}$ filter & $4,5,9$ & $3600,1200,40$ \\
\hline
\end{tabular}

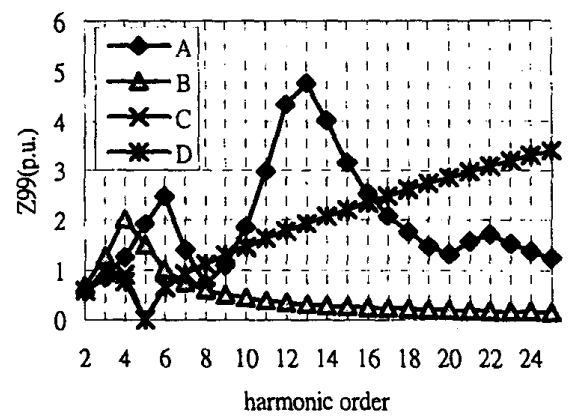

Fig. 1. Frequency scan of the driving-point impedance $z_{y y}$ at light load. 


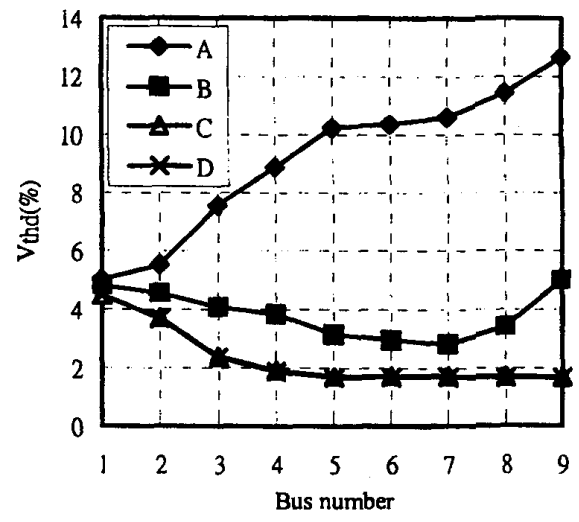

Fig. 2. The voltage total harmonic distortion of buses at light load.

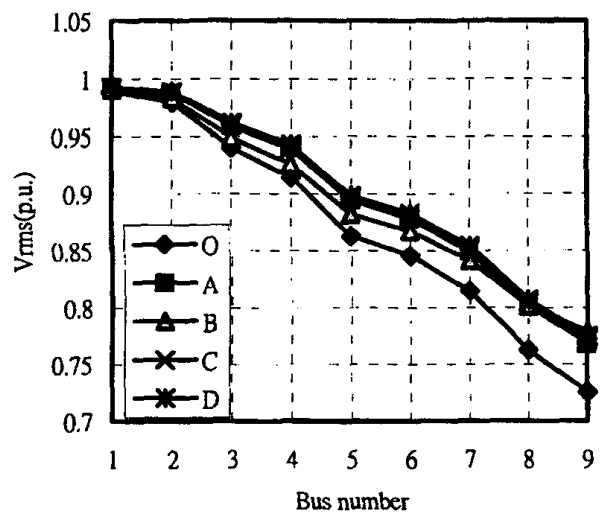

Fig. 3. The rms voltage values for each bus at peak load.

\section{CONCLUSIONS}

In this paper, we have proposed a refined differential evolution method to effectively determine the locations, types, and sizes of passive shunt harmonic filters to be installed on a radial distribution system. In our experience, control variables of $\mathrm{DE}$, e.g., $n p, F$ and $C R$ are easily tuned in the solving process. Simulation results show that both the RDE method and EP method are suitable for solving this problem. However, the RDE method can most likely provide the optimal solution within a reasonable time.

\section{REFERENCES}

[1] Comelia Kawann, Alexander E. Emanuel, "Passive Shunt Harmonic Filters For Low and Medium Voltage: A Cost Comparison Study," IEEE Transactions on Power Systems, Vol.1 1, No.4, November 1996, pp.1825-1831.

[2] Y. Baghzouz and S. Ertem, "Shunt capacitor sizing for Radial Distribution Feeders with Distorted Substation Voltages," IEEE Transactions on Power Delivery, Vol. 5, No.2, April 1990, pp.650657.
[3] M. Etezadi-Amoli and T. Florence, "Voltage and Current Harmonic Content of a Utility System- A Summary of 1120 Test Measurements," IEEE Transactions on Power Delivery, Vol. 5, No. 3, July 1990, pp. 1552-1557.

[4] A. E. Emanuel, J. A. Orr, D. Cyganski, and E. M. Gulachenski, "A Survey of Harmonic Voltages and Currents at Distribution Substations," IEEE Transactions on Power Delivery, Vol. 6, No. 4, October 1991, pp. 1883-1890.

[5] S. N. Govindarajan, M. D. Cox, and F. C. Berry, "Survey of Harmonic Levels on the Southwestern Electric Power Company System," IEEE Transactions on Power Delivery, Vol. 6, No. 4, October 1991, pp. 1869-1875.

[6] A. E. Emanuel, J. A. Or, D. Cyganski, and E. M. Gulachenski, "A Survey of Harmonic Voltages and Currents at Customer's Bus," IEEE Transactions on Power Delivery, Vol. 8, No. 1, January 1993, pp. $411-421$.

[7] R.F. Chu, Jin-Chen Wang, and Hsiao-Dong Chiang, "Strategic Planning of LC Compensators in Nonsinusoidal Distribution Systems," IEEE Transactions on Power Delivery, Vol.9, No.3, July1994, pp.1558-1563.

[8] Thomas H. Ortmeyer and Takashi Hiyama, "Distribution System Harmonic Filter Planning," IEEE Transactions on Power Delivery, Vol.11, No.4, October 1996, pp.2005-2012.

[9] Rainer Stom and Kenneth Price, "Differential Evolution A Simple and Efficient Heuristic for Global Optimization over Continuous Spaces," Joumal of Global Optimization, Vol. 11, 1997, pp.341-359.

\section{APPENDIX}

THREE-PHASE LOAD DATA

\begin{tabular}{|c|c|c|}
\hline Bus \# & $P(\mathrm{~kW})$ & $\mathrm{Q}(\mathrm{kvar})$ \\
\hline 1 & 1840 & 460 \\
2 & 980 & 340 \\
3 & 1790 & 446 \\
4 & 1598 & 1840 \\
5 & 1610 & 600 \\
6 & 780 & 110 \\
7 & 1150 & 60 \\
8 & 980 & 130 \\
9 & 1640 & 200 \\
\hline
\end{tabular}

FEEDER DATA AT $60 \mathrm{~Hz}$

\begin{tabular}{|c|c|c|c|}
\hline From Bus \# & To Bus \# & $R(\Omega)$ & $X(\Omega)$ \\
\hline 0 & 1 & 0.1233 & 0.4127 \\
1 & 2 & 0.0140 & 0.6051 \\
2 & 3 & 0.7463 & 1.2050 \\
3 & 4 & 0.6984 & 0.6084 \\
4 & 5 & 1.9831 & 1.7276 \\
5 & 6 & 0.9053 & 0.7886 \\
6 & 7 & 2.0552 & 1.1640 \\
7 & 8 & 4.7953 & 2.7160 \\
8 & 9 & 5.3434 & 3.0264 \\
\hline
\end{tabular}

Biography

Tien-Ting Chang was bom in Taitung, Taiwan on February 10, 1956. He received his B.S. degree from the Electrical Engineering Department of National Taiwan Institute of Technology in 1985, and his M.S. degree from National Taiwan University in 1988 . He is currently a Ph.D. student in the Electrical Engineering Department of National Taiwan University of Science and Technology. His research interests include electric power quality and distribution system planning.

Hong-Chan Chang was born in Taipei, Taiwan on March 5, 1959. He received his B.S., M.S., and Ph.D. degrees all from the Electrical Engineering Department of National Cheng Kung University in 1981, 1983 , and 1987, respectively. In August 1987 , he joined the National Taiwan University of Science and Technology where he is presently a professor in the Electrical Engineering Department. His major areas of research include powet system stability, control, and application of artificial intelligence to power systems. 